DOI: $10.3724 /$ SP.J.1218.2012.00519

\title{
四旋翼无人直升机鲁棒飞行控制
}

\author{
白永强 1,2 , 刘 昊 ${ }^{1}$, 石宗英 ${ }^{1}$, 钟宜生 ${ }^{1}$ \\ (1. 清华大学自动化系, 北京 $100084 ; 2$. 中国人民解放军 63880 部队)
}

\begin{abstract}
摘 要: 讨论了四旋翼无人直升机的飞行控制问题, 提出了一种鲁棒控制器设计方法. 该控制器由内环姿态控 制器和外环位置控制器两部分组成, 姿态控制器采用基于信号补偿的鲁棒控制方法, 位置控制器由经典的 PD 控制 实现. 将该控制器用于实验室自主研制的四旋翼无人直升机系统, 实现了室内悬停飞行. 实验结果验证了该控制方 法的有效性.
\end{abstract}

关键词: 四旋翼无人直升机; 飞行控制; 鲁棒补偿

中图分类号: TP182 文献标识码: A

文章编号: 1002-0446(2012)-05-0519-06

\section{Robust Flight Control of Quadrotor Unmanned Air Vehicles}

\author{
BAI Yongqiang ${ }^{1,2}$, LIU Hao ${ }^{1}$, SHI Zongying ${ }^{1}$, ZHONG Yisheng ${ }^{1}$ \\ (1. Department of Automation, Tsinghua University, Beijing 100084, China; \\ 2. No.63880 Unit of PLA, China)
}

\begin{abstract}
For quadrotor unmanned air vehicles (UAVs) flight control problem, a robust controller design approach is presented. The controller consists of two parts: an inner-loop attitude controller and an outer-loop position controller. The attitude controller is designed with robust control based on signal compensation. The position controller is realized with classical PD method. It is applied to a quadrotor UAV developed in our laboratory to realize indoor hovering. Experimental results demonstrate the effectiveness of this control approach.
\end{abstract}

Keywords: quadrotor UAV (unmanned air vehicle); flight control; robust compensation

\section{1 引言 (Introduction)}

近年来, 四旋翼无人直升机以其独特的外形、 结构与飞行方式逐渐成为国内外关注的热点 ${ }^{[1]}$. 与 常规布局的直升机相比, 四旋翼无人直升机机械结 构简单, 成本较低, 易于维护. 4 个螺旋桨的对称分 布, 使得四旋翼无人直升机的机动能力更强, 静态 盘旋的稳定性更好, 也更容易实现机型的微小型化. 四旋翼无人直升机特别适合在近地面环境（如室内、 城市和丛林等) 中执行监视、侦察等任务, 具有广阔 的应用前景 ${ }^{[2]}$.

对于四旋翼无人直升机的控制和系统实现，国 内外多家研究机构和高校进行了相关的研究 ${ }^{[3-8]}$, 有些还开发了四旋翼无人机平台 ${ }^{[6-10]}$. 在四旋翼无 人直升机的飞行控制上, 比较典型的方法有 PID 控 制 [11-12]、线性二次型最优调节控制 (LQR) ${ }^{[13]}$ 、滑 模控制 [14-15]、反步控制 (backstepping) ${ }^{[16]}$ 、动态逆 与 $\mathrm{H}_{\infty}$ 控制 [17] 等.
本实验室研制的四旋翼无人直升机基于航模 X650 的机体机械结构, 飞行控制系统和地面站 系统等均为自主研制. 其中, 飞行控制系统采用 TMS320F28335 高性能 DSP 作为飞控计算机, 传感 器系统包括三轴加速度计、三轴角速率陀螺、数字 罗盘、超声测距模块、GPS 和光流传感器等, 飞行 控制系统被安装在机体下方, 靠近质心位置, 如图 1 所示.

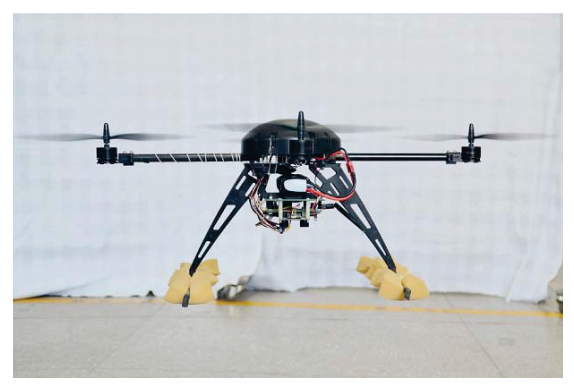

图 1 悬停飞行中的四旋翼无人直升机

Fig.1 Quadrotor UAV in hovering 
本文提出了一种基于鲁棒补偿的四旋翼无人直 升机控制方法, 并将之用于自主研制的四旋翼无人 直升机平台, 实现了稳定的室内定点悬停飞行。该 控制器由姿态控制器和位置控制器两部分组成: 姿 态控制采用了基于信号补偿的鲁棒控制 [18-19]，位置 控制则采用经典的 PD 控制. 这种方法控制结构清 晰, 控制器参数易于调节, 便于工程实现. 飞行实验 结果验证了所提控制方法的有效性.

\section{2 动力学模型 (Dynamics model)}

以 $E=\left\{X_{\mathrm{e}} Y_{\mathrm{e}} Z_{\mathrm{e}}\right\}$ 表示惯性坐标系, $B=\left\{X_{\mathrm{b}} Y_{\mathrm{b}} Z_{\mathrm{b}}\right\}$ 表示机体坐标系, $\boldsymbol{\Phi}=[\varphi, \theta, \psi]^{\mathrm{T}}$ 表示欧拉角, 则 从惯性坐标系到机体坐标系的旋转矩阵 $\boldsymbol{R}: E \rightarrow B$ 为

$$
\boldsymbol{R}=\left[\begin{array}{ccc}
C_{\theta} C_{\psi} & C_{\psi} S_{\varphi} S_{\theta}+C_{\varphi} S_{\psi} & S_{\varphi} S_{\psi}-C_{\varphi} C_{\psi} S_{\theta} \\
-C_{\theta} S_{\psi} & C_{\varphi} C_{\psi}-S_{\varphi} S_{\theta} S_{\psi} & C_{\varphi} S_{\theta} S_{\psi}+C_{\psi} S_{\varphi} \\
S_{\theta} & -C_{\theta} S_{\varphi} & C_{\varphi} C_{\theta}
\end{array}\right]
$$

为表示方便, 简记 $C_{\theta}=\cos \theta, S_{\theta}=\sin \theta$, 下文 $T_{\theta}=$ $\tan \theta$ 类似.

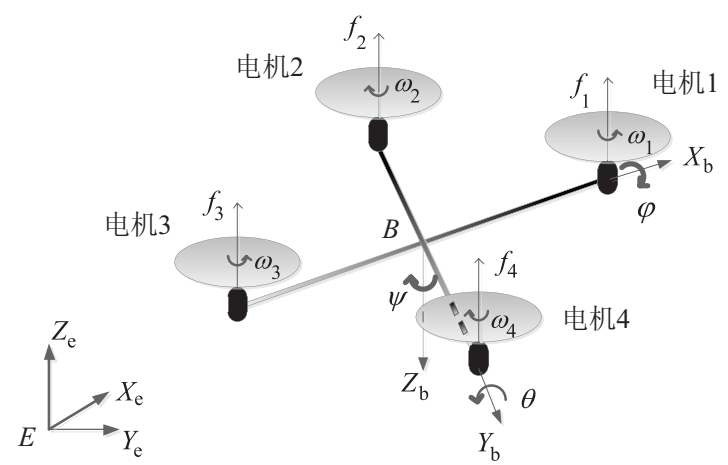

图 2 四旋翼无人直升机动力学示意图

Fig.2 Schematic of quadrotor UAV dynamics

如图 2 所示, 四旋翼无人直升机的 $1 、 3$ 旋翼为 逆时针旋转, 而 $2 、 4$ 旋翼为顺时针旋转, 因此当 4 个电机转速一致且升力之和等于机体自重时, 无人 机可以保持悬停, 当升力同时增加或减少时, 无人 机可上升或下降; 保持 4 个旋翼升力和与无人机自 重相等，1、3 旋翼增速 (减速) 且 $2 、 4$ 旋翼减速 (增 速), 由于扭矩差的作用, 无人机可实现偏航运动; 旋翼 1 增速 (减速)、旋翼 3 减速 (增速) 而旋翼 2、4 速度维持不变, 无人机可实现关于 $Y_{\mathrm{b}}$ 轴的俯仰运 动, 类似地也可实现关于 $X_{\mathrm{b}}$ 轴的滚转运动. 将以 上的基本动作进行组合, 无人机可实现各种复杂运 动.

根据牛顿运动定律与欧拉方程, 四旋翼无人直
升机的动力学方程可表示为 [20]

$$
\begin{gathered}
\boldsymbol{F}=m \ddot{\boldsymbol{P}} \\
\boldsymbol{M}=\frac{\mathrm{d} \boldsymbol{H}}{\mathrm{d} t}
\end{gathered}
$$

其中, $\boldsymbol{F}$ 是无人机受到的外部合力, $m$ 是无人机的 质量, $\boldsymbol{M}$ 是机体转动力矩, $\boldsymbol{H}$ 是惯性坐标系下的机 体角动量. 以 $l$ 表示电机转轴到机体中心的距离, $J_{\mathrm{r}}$ 表示电机的惯量, $f_{i}(i=1,2,3,4)$ 表示第 $i$ 个旋翼 提供的升力, $b$ 和 $d$ 分别表示旋翼的升力和阻力系 数, $\boldsymbol{J}$ 表示机体的惯性矩阵, $\boldsymbol{K}_{\mathrm{dm}}$ 表示转动阻力矩系 数, $\boldsymbol{K}_{\mathrm{dt}}$ 表示平动阻力系数, 根据四旋翼无人机的结 构特点, $\boldsymbol{J} 、 \boldsymbol{K}_{\mathrm{dm}}$ 和 $\boldsymbol{K}_{\mathrm{dt}}$ 都可以表示为对角阵:

$$
\begin{aligned}
& \boldsymbol{J}=\operatorname{diag}\left(I_{x}, \quad I_{y}, I_{z}\right) \\
& \boldsymbol{K}_{\mathrm{dm}}=\operatorname{diag}\left(K_{\mathrm{dm} x}, K_{\mathrm{dm} y}, \quad K_{\mathrm{dm} z}\right) \\
& \boldsymbol{K}_{\mathrm{dt}}=\operatorname{diag}\left(K_{\mathrm{dt} x}, K_{\mathrm{dt} y}, \quad K_{\mathrm{dt} z}\right)
\end{aligned}
$$

以 $\boldsymbol{P}=[x, y, z]^{\mathrm{T}}$ 表示惯性坐标系 $E$ 下无人机 质心的位置, $\boldsymbol{\Omega}=[p, q, r]^{\mathrm{T}}$ 表示机体坐标系 $B$ 下 绕 3 个轴的旋转角速度, 则有 ${ }^{[5]}$ :

$$
\begin{aligned}
& \ddot{x}=\left(C_{\varphi} C_{\psi} S_{\theta}+S_{\varphi} S_{\psi}\right) \frac{1}{m} \sum_{i=1}^{4} f_{i}-\frac{K_{\mathrm{dt} x}}{m} \dot{x} \\
& \ddot{y}=\left(C_{\varphi} S_{\psi} S_{\theta}-S_{\varphi} C_{\psi}\right) \frac{1}{m} \sum_{i=1}^{4} f_{i}-\frac{K_{\mathrm{dt} y}}{m} \dot{y} \\
& \ddot{z}=\left(C_{\theta} C_{\varphi}\right) \frac{1}{m} \sum_{i=1}^{4} f_{i}-\frac{K_{\mathrm{dt} z}}{m} \dot{z}-g \\
& \dot{p}=q r\left(\frac{I_{y}-I_{z}}{I_{x}}\right)+\frac{l}{I_{x}} b\left(\omega_{4}^{2}-\omega_{2}^{2}\right)-\frac{K_{\mathrm{dm} x}}{I_{x}} p+ \\
& \frac{J_{\mathrm{r}}}{I_{x}} q\left(\omega_{1}+\omega_{3}-\omega_{2}-\omega_{4}\right) \\
& \dot{q}=p r\left(\frac{I_{z}-I_{x}}{I_{y}}\right)+\frac{l}{I_{y}} b\left(\omega_{3}^{2}-\omega_{1}^{2}\right)-\frac{K_{\mathrm{dm} y}}{I_{y}} q+ \\
& \frac{J_{\mathrm{r}}}{I_{y}} p\left(\omega_{2}+\omega_{4}-\omega_{1}-\omega_{3}\right) \\
& \dot{r}=p q\left(\frac{I_{x}-I_{y}}{I_{z}}\right)+\frac{d}{I_{z}}\left(\omega_{1}^{2}+\omega_{3}^{2}-\omega_{4}^{2}-\omega_{2}^{2}\right)- \\
& \frac{K_{\mathrm{dm} z}}{I_{z}} r
\end{aligned}
$$

这里 $f_{i}=b \omega_{i}^{2}, \omega_{i}$ 是第 $i$ 个旋翼的旋转角速度. 方程 (3) 和方程 (4) 分别描述了四旋翼无人机的质心平移 运动和机体转动运动. 欧拉角速度与机体坐标系下 的角速度间存在如下关系:

$$
\left[\begin{array}{c}
\dot{\varphi} \\
\dot{\theta} \\
\dot{\psi}
\end{array}\right]=\left[\begin{array}{ccc}
1 & S_{\varphi} T_{\theta} & C_{\varphi} T_{\theta} \\
0 & C_{\varphi} & -S_{\varphi} \\
0 & S_{\varphi} / C_{\theta} & C_{\varphi} / C_{\theta}
\end{array}\right]\left[\begin{array}{c}
p \\
q \\
r
\end{array}\right]
$$


当四旋翼无人机在室内低速飞行或者悬停时, 其所受到的空气阻力可以忽略不计, 定义向量 $\boldsymbol{U}=$ $\left[u_{1}, u_{2}, u_{3}, u_{4}\right]^{\mathrm{T}}$ 如下:

$$
\begin{aligned}
& u_{1}=b\left(\omega_{1}^{2}+\omega_{2}^{2}+\omega_{3}^{2}+\omega_{4}^{2}\right) \\
& u_{2}=b\left(\omega_{2}^{2}-\omega_{4}^{2}\right) \\
& u_{3}=b\left(\omega_{1}^{2}-\omega_{3}^{2}\right) \\
& u_{4}=d\left(\omega_{1}^{2}+\omega_{3}^{2}-\omega_{4}^{2}-\omega_{2}^{2}\right)
\end{aligned}
$$

则可以得到以下简化模型:

$$
\begin{aligned}
\ddot{x} & =\left(C_{\varphi} C_{\psi} S_{\theta}+S_{\varphi} S_{\psi}\right) \frac{1}{m} u_{1} \\
\ddot{y} & =\left(C_{\varphi} S_{\psi} S_{\theta}-S_{\varphi} C_{\psi}\right) \frac{1}{m} u_{1} \\
\ddot{z} & =\left(C_{\theta} C_{\varphi}\right) \frac{1}{m} u_{1}-g \\
\ddot{\varphi} & =\dot{\theta} \dot{\psi}\left(\frac{I_{y}-I_{z}}{I_{x}}\right)+\frac{l}{I_{x}} u_{2} \\
\ddot{\theta} & =\dot{\varphi} \dot{\psi}\left(\frac{I_{z}-I_{x}}{I_{y}}\right)+\frac{l}{I_{y}} u_{3} \\
\ddot{\psi} & =\dot{\varphi} \dot{\theta}\left(\frac{I_{x}-I_{y}}{I_{z}}\right)+\frac{1}{I_{z}} u_{4}
\end{aligned}
$$

在下一节的控制器设计中, 将以向量 $\boldsymbol{U}$ 作为四 旋翼无人直升机的控制输入.

\section{3 控制器设计（Controller design）}

从四旋翼无人机的动力学模型可以发现, 四旋 翼的运动可以分解为质心移动和机体转动. 惯性坐 标系下的机体质心移动依赖于机体侧倾获取的侧向 加速度, 因此通常的四旋翼控制都通过嵌套的内外 环控制实现, 其中内环是姿态环, 而外环是位置环. 图 3 给出了系统的控制结构示意图, 其中 $\psi_{\mathrm{r}}, \boldsymbol{P}_{\mathrm{r}}, \boldsymbol{\Phi}_{\mathrm{r}}$ 分别是偏航角、位置和欧拉角的参考值.

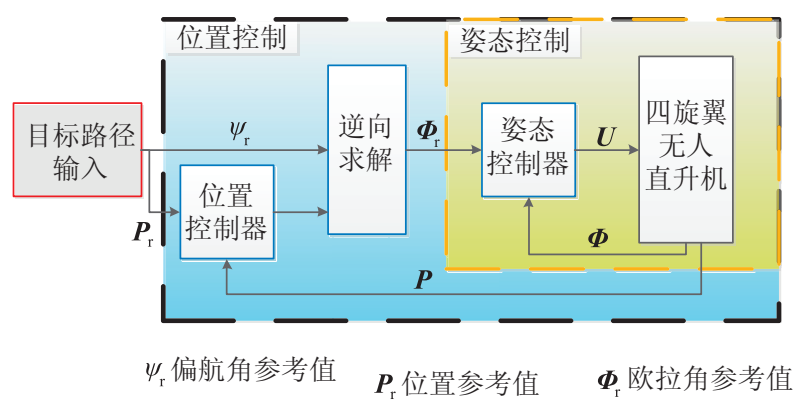

图 3 控制结构示意图

Fig.3 Structure of control system

\section{1 姿态控制}

姿态控制是四旋翼控制的核心，不仅是因为姿 态控制的结果直接影响飞行品质, 更因为它是位置 控制的前提. 设计姿态控制器如图 4, 该控制器由线
性标称控制器和鲁棒补偿器两部分组成, 其中线性 标称控制器基于线性标称模型设计, 使标称闭环系 统获得理想的性能; 鲁棒补偿器用来抵消外部有界 扰动、未建模的非线性因素及参数不确定性等带来 的不利影响.

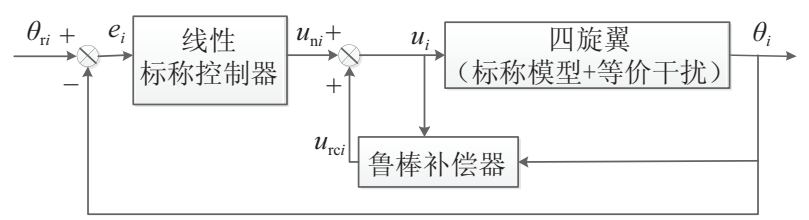

图 4 姿态控制器

Fig.4 The schematic diagram of the attitude control

根据方程 (7), 可得四旋翼无人机平衡点附近的 姿态角变化的线性化模型为

$$
\begin{aligned}
\ddot{\varphi} & =\frac{l}{I_{x}} u_{2} \\
\ddot{\theta} & =\frac{l}{I_{y}} u_{3} \\
\ddot{\psi} & =\frac{1}{I_{z}} u_{4}
\end{aligned}
$$

将其作为四旋翼无人机姿态控制的标称模型. 由于方程 (8) 中 3 个姿态角的运动方程表达式是类 似的, 这里仅以俯仰角为例来讨论姿态控制器的设 计问题. 为描述方便, 将俯仰角的线性标称模型表 示为

$$
\ddot{\theta}=a u_{\mathrm{n} 3}
$$

其中, $a=\frac{l}{I_{y}}$ 是一个正常数, $u_{\mathrm{n} 3}$ 是控制输入 $u_{3}$ 的标 称值. 在频率域可表示为

$$
\theta(s)=\frac{a}{s^{2}} u_{\mathrm{n} 3}(s)=G_{0}(s) u_{\mathrm{n} 3}(s)
$$

其中 $\theta(s)$ 表示 $\theta(t)$ 的拉氏变换, 其他类似. 设计线 性标称控制器:

$$
u_{\mathrm{n} 3}(s)=\frac{N_{y}(s)}{D_{u}(s)} e(s)
$$

其中, $N_{y}(s)=\alpha s+\beta, D_{u}(s)=s+\gamma, e(s)=\theta_{\mathrm{r}}(s)-$ $\theta(s) . \alpha 、 \beta$ 和 $\gamma$ 均是正常数, $N_{y}(s)$ 和 $D_{u}(s)$ 满足:

$$
D_{\mathrm{m}}(s) H(s)=s^{2} D_{u}(s)-a N_{y}(s)
$$

这里 $D_{\mathrm{m}}(s)=s^{2}+a_{\mathrm{m}} s+b_{\mathrm{m}}$ 是标称闭环系统的理想 特征多项式, $H(s)=s+h_{0}$ 是赫尔维兹多项式.

上述标称线性控制器的设计基于标称模型, 然 而标称模型与实际系统模型间是有差异的. 本文将 实际系统和标称模型之间的差异对系统的影响视为 等价干扰的作用, 定义 $d_{\theta}$ 为等价干扰, 它包括标称 模型中忽略的与其它轴之间的耦合、非线性项、高 次项, 以及有界外部干扰、参数摄动等不确定性. 下 
面设计鲁棒补偿器, 使其产生的鲁棒补偿信号能够 抑制等价干扰对闭环系统的影响 ${ }^{[18]}$.

实际系统的俯仰角模型可以表示为

$$
\ddot{\theta}=a u_{3}+d_{\theta}
$$

其中控制输入 $u_{3}$ 由标称控制输入 $u_{\mathrm{n} 3}$ 和鲁棒补偿输 入 $u_{\mathrm{rc} 3}$ 两部分组成:

$$
u_{3}=u_{\mathrm{n} 3}+u_{\mathrm{rc} 3}
$$

设计鲁棒补偿器如下 ${ }^{[19]}$ :

$$
u_{\mathrm{rc} 3}(s)=-\frac{1}{a} F_{\theta}(s) d_{\theta}(s)
$$

这里 $F_{\theta}(s)=f_{\theta} g_{\theta} /\left(s+f_{\theta}\right)\left(s+g_{\theta}\right)$, 其中 $s$ 是微分算 子, $f_{\theta}$ 和 $g_{\theta}$ 是正常数. 当 $f_{\theta}$ 和 $g_{\theta}$ 足够大时, $u_{\mathrm{rc} 3}(s)$ 就接近 $-d_{\theta}(s) / a$, 从而抑制 $d_{\theta}$ 的影响. $u_{\mathrm{rc} 3}$ 可以进 一步表示为

$$
u_{\mathrm{rc} 3}(s)=-\frac{1}{a} \frac{F_{\theta}(s)}{1-F_{\theta}(s)}\left(s^{2} \theta(s)-a u_{\mathrm{n} 3}(s)\right)
$$

联立方程 (11)、(14) 和 (16), 即可得到俯仰角的控制 输入 $u_{3}{ }^{[21]}$.

用同样的方法, 可以实现横滚角与偏航角的控 制. 在此姿态控制器的设计过程中, 线性标称控制 器和鲁棒补偿器是依次设计的, 因此在系统实现中, 可以先确定标称控制器, 然后再根据系统特性单向 调节 $f_{j}$ 和 $g_{j}(j=\theta, \varphi, \psi)$, 实现鲁棒补偿.

\section{2 位置控制}

在实现姿态控制的基础上，设计四旋翼无人直 升机的位置控制.

由方程 (7) 得到四旋翼无人直升机在室内低速 飞行或悬停时的位置变化简化模型:

$$
\begin{aligned}
\ddot{x} & =\sin \theta \frac{1}{m} u_{1} \\
\ddot{y} & =-\sin \varphi \frac{1}{m} u_{1} \\
\ddot{z} & =\cos \theta \cos \varphi \frac{1}{m} u_{1}-g
\end{aligned}
$$

其空间位置控制可以分为高度控制和水平位置 控制. 设计高度控制器如下:

$$
u_{1}=\frac{1}{\cos \varphi \cos \theta}\left(K_{\mathrm{p}}^{z}\left(z_{\mathrm{r}}-z\right)+K_{\mathrm{d}}^{z}\left(\dot{z}_{\mathrm{r}}-\dot{z}\right)+m g\right)
$$

该控制器基于带非线性补偿的 $\mathrm{PD}$ 控制, 其中 $z$ 是无 人直升机飞行高度, $z_{\mathrm{r}}$ 是高度的参考值. 项补偿了机体在俯仰或滚转运动时的升力损失.
由于四旋翼无人直升机的对称结构, 仅以 $X_{\mathrm{e}}$ 轴 向运动为例考虑水平位置的控制, 设计 $\mathrm{PD}$ 控制器:

$$
\begin{aligned}
& \theta_{\mathrm{r}}=\arcsin \left(K_{\mathrm{p}}^{x}\left(x_{\mathrm{r}}-x\right)+K_{\mathrm{d}}^{x}\left(\dot{x}_{\mathrm{r}}-\dot{x}\right)\right) \\
& \dot{\theta}_{\mathrm{r}}=\frac{K_{\mathrm{p}}^{x} \dot{x}+K_{\mathrm{d}}^{x} \ddot{x}}{\sqrt{1-K_{\mathrm{p}}^{x 2} x^{2}-2 K_{\mathrm{p}}^{x} K_{\mathrm{d}}^{x} x \dot{x}-K_{\mathrm{d}}^{x 2} \dot{x}^{2}}}
\end{aligned}
$$

该控制器的输出即为内环姿态控制器的输入, $\theta_{\mathrm{r}}$ 和 $\dot{\theta}_{\mathrm{r}}$ 分别是俯仰角度和俯仰角速度参考值.

\section{4 实验验证 (Experiment validation)}

将上述设计的鲁棒控制器用于自主研制的四旋 翼无人直升机, 采用测量精度达到厘米级的光流传 感器作为室内飞行的位置与速度检测手段 (光流传 感器安装于机体下方, 视线垂直于地面), 进行了定 点悬停控制实验, 并将鲁棒补偿控制的姿态实验结 果和 PID 控制的姿态实验结果作以比较.

在姿态控制实验过程中, 将 3 个姿态角的理想 值均设为 $0^{\circ}$, 分别采用 PID 控制与鲁棒补偿控制进 行了实验, 图 5 和图 6 分别给出了两种控制器作用

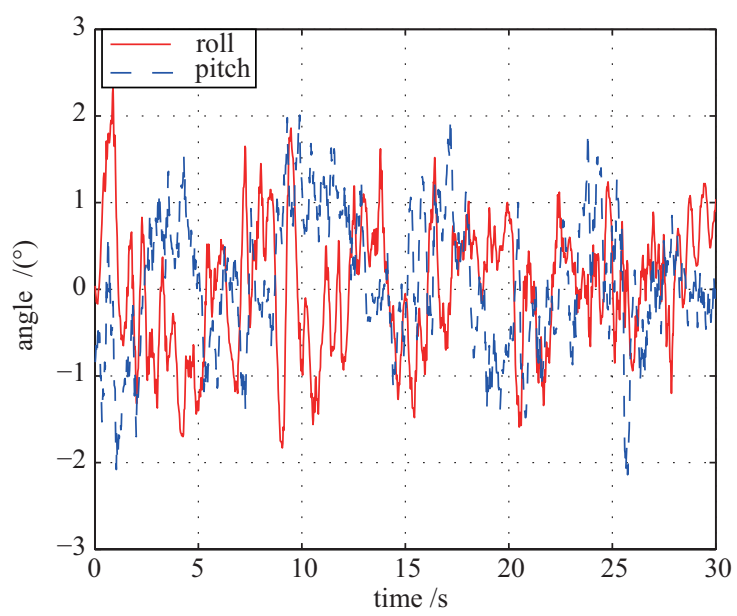

图 5 基于 PID 控制的横滚、俯仰角响应曲线

Fig.5 Response of roll and pitch under PID control

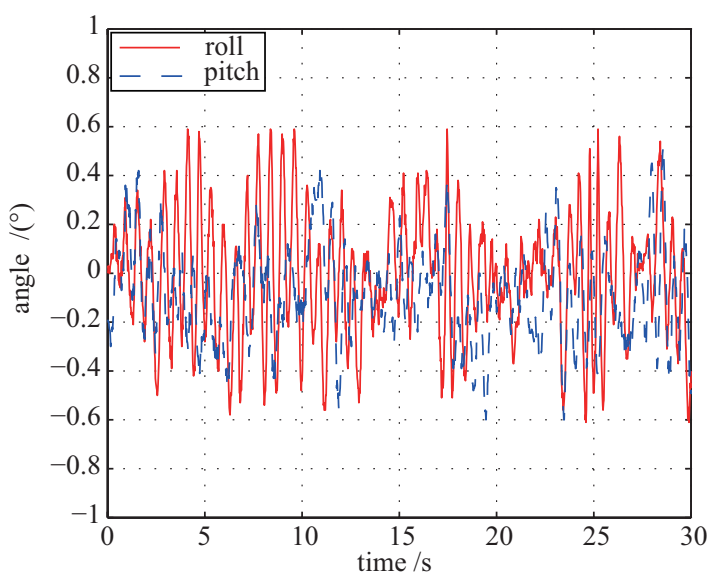

图 6 基于鲁棒补偿控制的横滚、俯仰角响应曲线 Fig.6 Response of roll and pitch under robust control 
下的横滚、俯仰角响应曲线. 从图中可以看到, PID 控制器可以将姿态角控制在 $\pm 2^{\circ}$ 以内, 而鲁棒补偿 控制器可以将姿态角控制在 $\pm 0.6^{\circ}$ 以内, 控制精度 较高.

将鲁棒补偿姿态控制器结合 PD 位置控制器应 用于系统平台进行定点悬停实验, 此时偏航角的理

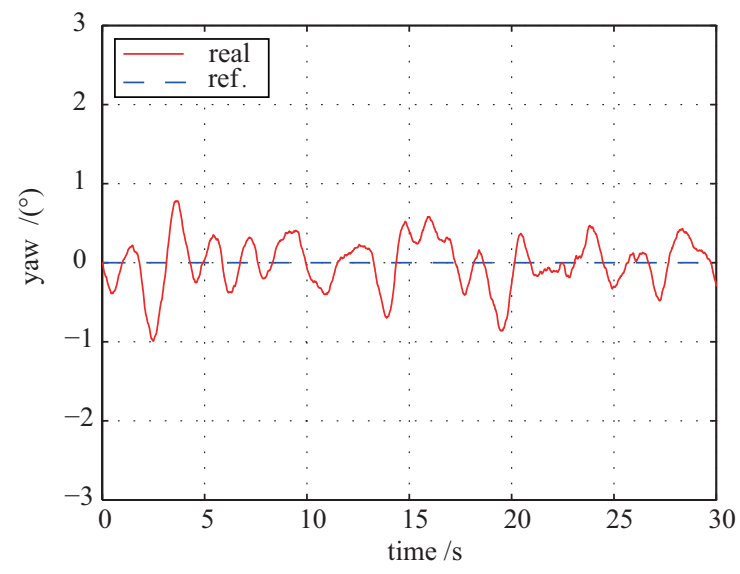

图 7 偏航角响应曲线

Fig.7 Response of yaw angle

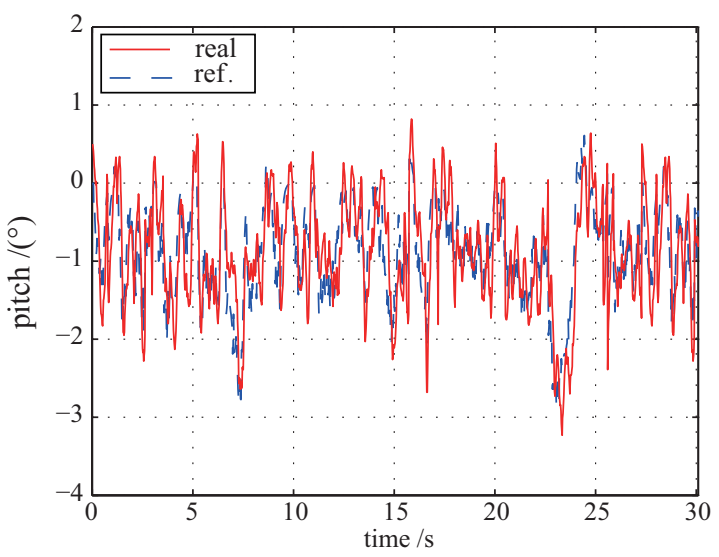

图 8 俯仰角响应曲线

Fig.8 Response of pitch angle

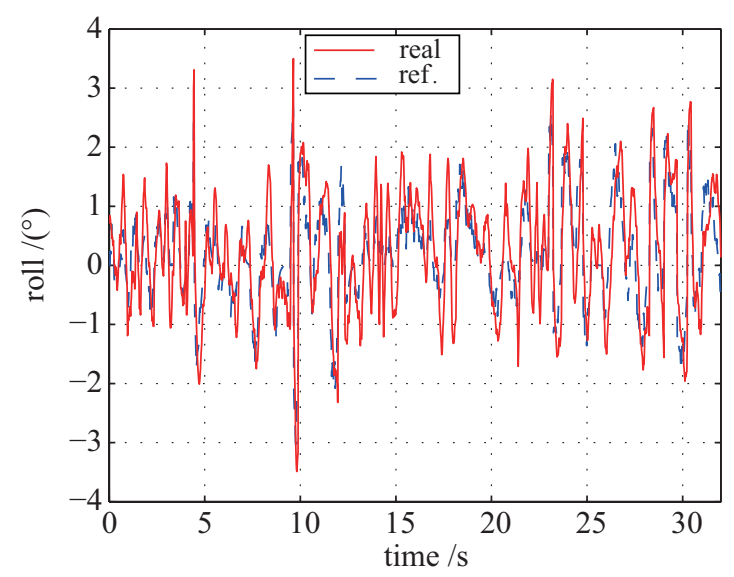

图 9 横滚角响应曲线

Fig.9 Response of roll angle
想值设为 $0^{\circ}$, 其响应曲线如图 7 所示. 结果显示偏 航角的误差在 $0.8^{\circ}$ 以内, 其均方根误差是 $0.3226^{\circ}$. 由上节控制器设计可知, 水平位置控制器输出的 角度值被作为俯仰角和横滚角控制的参考输入, 图 8、9 分别给出了俯仰角和横滚角的响应曲线. 从实 验结果可以看到, 俯仰角和横滚角可以快速响应输 入值的变化, 跟踪误差小于 $\pm 1^{\circ}$, 均方根误差分别 是 $0.4728^{\circ}$ 和 $0.6087^{\circ}$.

在其他机构的类似实验中，澳大利亚的 X-4 四 旋翼无人直升机采用离散 PID 控制方法, 室内悬停 飞行的姿态角控制误差为 $\pm 1^{\circ[6]}$. 美国斯坦福大学 的 STARMAC 四旋翼无人直升机设计了带角加速度 反馈的 PID 姿态控制器, 姿态角跟踪的均方根误差 为 $0.65^{\circ}$, 在机动飞行 $\left( \pm 15^{\circ}\right)$ 时能将跟踪误差控制 在 $\pm 3^{\circ}$ 以内 ${ }^{[7]}$. 比较可知, 本文的控制方法是有效 的.

图 10 和图 11 是四旋翼悬停飞行的高度曲线 和水平位置运动轨迹, 高度控制设置的理想值为

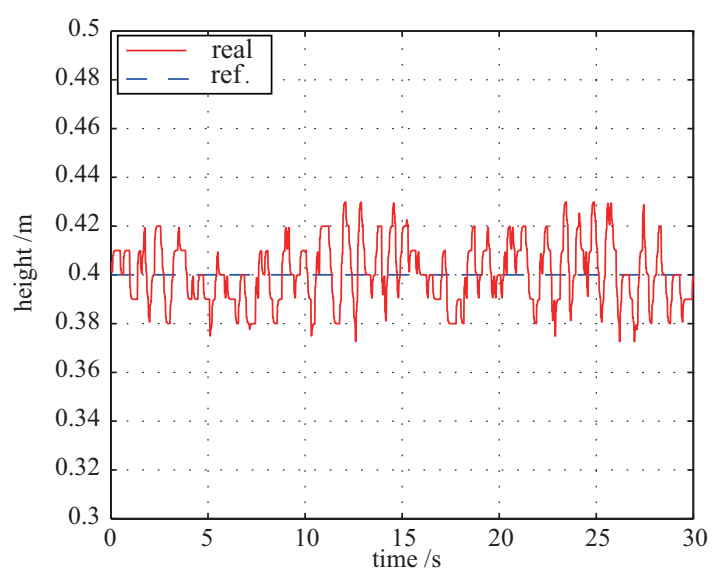

图 10 悬停飞行中的高度曲线

Fig.10 Altitude in hovering

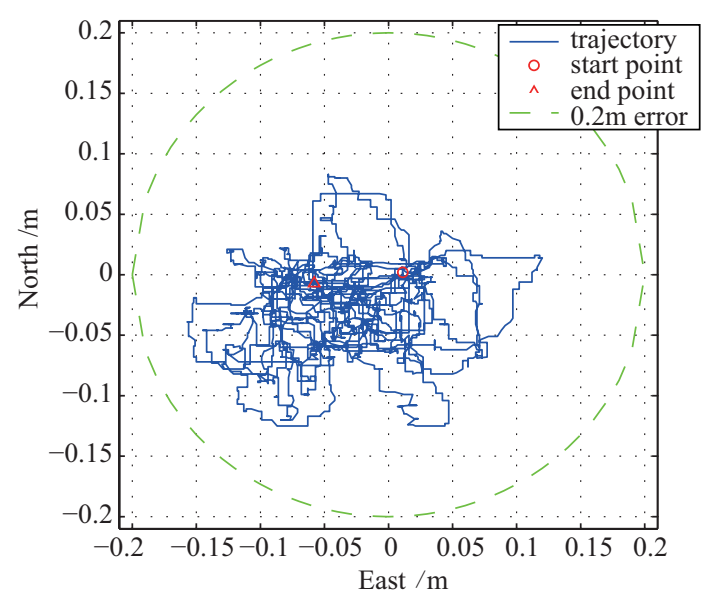

图 11 水平位置轨迹曲线

Fig.11 Horizontal position trajectory 
$0.4 \mathrm{~m}$, 结果显示飞行器的飞行高度可以维持在 $0.38 \mathrm{~m} \sim 0.43 \mathrm{~m}$ 之间, 控制误差小于 $3 \mathrm{~cm}$, 水平 位置可以控制在 $\pm 0.2 \mathrm{~m}$ 的范围内.

\section{5 结论 (Conclusion)}

针对四旋翼无人直升机的飞行控制问题，本文 提出了一种基于鲁棒补偿的控制器设计方法, 该控 制器由鲁棒姿态控制器与 PD 位置控制器组成, 将 其应用于自主研制的四旋翼无人直升机上, 实现了 室内自主悬停. 实验结果验证了该鲁棒补偿控制方 法的有效性.

在未来的工作中，将会在位置控制中尝试鲁棒 补偿等其他控制方法, 以实现精确的轨迹跟踪与航 迹飞行.

\section{参考文献（References）}

[1] 岳基隆, 张庆杰, 朱华勇. 微小型四旋翼无人机研究进展及 关键技术浅析 [J]. 电光与控制, 2010, 17(10): 46-52.

Yue J L, Zhang Q J, Zhu H Y. Research progress and key technologies of micro quad-rotor UAVs[J]. Electronics Optics and Control, 2010, 17(10): 46-52.

[2] 聂博文, 马宏绪, 王剑, 等. 微小型四旋翼飞行器的研究现 状与关键技术 $[\mathrm{J}]$. 电光与控制, 2007, 14(6): 113-117.

Nie B W, Ma H X, Wang J, et al. Study on actualities and critical technologies of micro/mini quadrotor[J]. Electronics Optics and Control, 2007, 14(6): 113-117.

[3] Altug E, Ostrowski J P, Taylor C J. Quadrotor control using dual camera visual feedback[C]//IEEE International Conference on Robotics and Automation. Piscataway, NJ, USA: IEEE, 2003: 4294-4299.

[4] Valenti M, Bethke B, Fiore G, et al. Indoor multi-vehicle flight testbed for fault detection, isolation, and recovery[C]//Proceedings of the AIAA Guidance, Navigation and Control Conference and Exhibit. Reston, VA, USA: AIAA, 2006.

[5] Bouabdallah S, Siegwart R. Full control of a quadrotor[C]//IEEE/RSJ International Conference on Intelligent Robots and Systems. Piscataway, NJ, USA: IEEE, 2007: 153158.

[6] Pounds P, Mahony R, Corke P. Modelling and control of a large quadrotor robot[J]. Control Engineering Practice, 2010, 18(7): 691-699.

[7] Hoffmann G M, Huang H M, Waslander S L, et al. Precision flight control for a multi-vehicle quadrotor helicopter testbed[J]. Control Engineering Practice, 2011, 19(9): 1023-1036.

[8] Alexis K, Nikolakopoulos G, Tzes A. Switching model predictive attitude control for a quadrotor helicopter subject to atmospheric disturbances[J]. Control Engineering Practice, 2011, 19(10): 1195-1207.

[9] Mellinger D, Kumar V. Minimum snap trajectory generation and control for quadrotors[C]//IEEE International Conference on Robotics and Automation. Piscataway, NJ, USA: IEEE, 2011: 2520-2525.

[10] 刘晓杰. 基于视觉的微小型四旋翼飞行器位姿估计研究与 实现 [D]. 长春: 吉林大学, 2009.
Liu X J. Study and implementation of position and attitude estimation for four-rotor mini rotorcraft based on vision[D]. Changchun, China: Jilin University, 2009.

[11] Salih A L, Moghavvemi M, Mohamed H A F, et al. Modelling and PID controller design for a quadrotor unmanned air vehicle[C]//IEEE International Conference on Automation, Quality and Testing, Robotics. Piscataway, NJ, USA: IEEE, 2010.

[12] 宿敬亚, 樊鹏辉, 蔡开元. 四旋翼飞行器的非线性 PID 姿态 控制 [J]. 北京航空航天大学学报, 2011, 37(9): 1054-1058.

Su J Y, Fan P H, Cai K Y. Attitude control of quadrotor aircraft via nonlinear PID[J]. Journal of Beijing University of Aeronautics and Astronautics, 2011, 37(9): 1054-1058.

[13] Bouabdallah S, Noth A, Siegwart R. PID vs LQ control techniques applied to an indoor micro quadrotor[C]//EEE/RSJ International Conference on Intelligent Robots and Systems. Piscataway, NJ, USA: IEEE, 2004: 2451-2456.

[14] Benallegue A, Mokhtari A, Fridman L. Feedback linearization and high order sliding mode observer for a quadrotor UAV[C]//International Workshop on Variable Structure Systems. Piscataway, NJ, USA: IEEE, 2006: 365-372.

[15] Waslander S L, Hoffmann G M, Jang J S, et al. Multi agent quadrotor testbed control design: Integral sliding-mode vs. reinforcement learning[C]//IEEE/RSJ International Conference on Intelligent Robotics and Systems. Piscataway, NJ, USA: IEEE, 2005: 468-473.

[16] Bouabdallah S, Siegwart R. Backstepping and sliding mode techniques applied to an indoor micro quadrotor[C]//IEEE International Conference on Robotics and Automation. Piscataway, NJ, USA: IEEE, 2005: 2247-2252.

[17] Raffo G V, Ortega M G, Rubio F R. An integral predictive/nonlinear $\mathrm{H}_{\infty}$ control structure for a quadrotor helicopter[J]. Automatica, 2010, 46(1): 29-39.

[18] Zhong Y S. Robust output tracking control of SISO plants with multiple operating points and with parametric and unstructured uncertainties[J]. International Journal of Control, 2002, 75(4): 219-241.

[19] 钟宜生. 基于信号补偿的鲁棒控制方法 [J]. 清华大学学报: 自然科学版, 2003, 43(4): 542-563.

Zhong Y S. Robust control based on signal compensation[J]. Journal of Tsinghua University: Science and Technology, 2003, 43(4): 542-563.

[20] Bouabdallah S, Murrieri P, Siegwart R. Design and control of an indoor micro quadrotor[C]//IEEE International Conference on Robotics and Automation. Piscataway, NJ, USA: IEEE, 2004: 4393-4398.

[21] Liu H, Yu Y, Lu G, et al. Robust LQR attitude control of a 3DOF lab helicopter[C]//29th Chinese Control Conference. Piscataway, NJ, USA: IEEE, 2010: 529-534.

\section{作者简介:}

白永强 (1986-), 男, 硕士生, 助理工程师. 研究领域: 四 旋翼无人直升机的控制与系统实现.

石宗英 (1969-), 女, 博士, 副教授. 研究领域: 无人直升 机自主导航与控制, 移动机器人定位与 SLAM, 机 器人控制.

钟宜生 (1958-), 男, 博士, 教授. 研究领域: 鲁棒控制, 非线性控制. 